\title{
A novel mild variant of osteogenesis imperfecta type I caused by a Gly1088Glu mutation in COL1A1
}

\author{
XIN-YI XIA, WEI-WEI LI, NA LI, QIU-YUE WU, YING-XIA CUI and XIAO-JUN LI \\ Institute of Laboratory Medicine, Jinling Hospital, Nanjing University School of Medicine, Nanjing 210002, P.R. China
}

Received August 30, 2013; Accepted March 4, 2014

DOI: $10.3892 / \mathrm{mmr} .2014 .2084$

\begin{abstract}
Osteogenesis imperfecta (OI), also known as brittle bone disease, characterized by multiplicative osteopsathyrosis, blue sclera, dentinogenesis imperfecta and mild audition, is a rare inherited connective tissue disease. There are seven types of OI, I to VII, among which type I-IV are relatively common and associated with type I collagen. Defects in type I collagen synthesis or structure are responsible for the majority of clinical OI cases since collagen is the major matrix protein of all connective tissues. Type I collagen consists of two pro- $\alpha 1$ chains and one pro- $\alpha 2$ chain, which are encoded by two genes, COL1A1 and COL1A2, respectively. The two subunits have a Gly-X-Y repeat domain, of which glycine is highly conserved in the majority of species. Point mutations on these sites appear to trigger OI. In the current study, a heterozygous mutation, c.3263G>A, p.Gly1088Glu, was identified in the Gly-X-Y domain of type I collagen in an affected individual with type I OI. A lethal phenotype with the p.Gly1088Ala mutation was observed at the same site as the current findings. This suggests that variant characteristics of the substitution for Gly may trigger a varying degree of OI from lethal to mild, even when the mutation occurs at the same site. It is hypothesized that the study may provide insight into the phenotype-genotype association and may assist, not only in the clinical diagnosis, but also in investigating the mechanism of collagen-associated diseases.
\end{abstract}

\section{Introduction}

Osteogenesis imperfecta (OI), also known as brittle bone disease, characterized by increased bone fragility and low bone mass, is a rare inherited connective tissue disease $(1,2)$, mainly with autosomal dominant inheritance. The incidence of OI is 1:10,000 and the capital clinical manifestations of OI are multiplicative osteopsathyrosis, blue sclera, dentinogenesis

Correspondence to: Professor Xiao-Jun Li, Institute of Laboratory Medicine, Jinling Hospital, Nanjing University School of Medicine, 305 East Zhongshan Road, Nanjing 210002, P.R. China

E-mail: lixiaojun1962@gmail.com

Key words: osteogenesis imperfect type I, COL1Al gene, point mutation, Gly-X-Y domain imperfecta and mild audition (3). The traditional classification is based on clinical and radiographic criteria, and the mode of inheritance findings include type I (MIM \#166200), II (MIM \#166210), III (MIM \#259420) and IV (MIM \#166220) (4,5). Their phenotypic presentation varies from mild to lethal, of which type I is the mildest with the highest incidence rate.

More than $90 \%$ patients with OI types I-IV have autosomal dominant mutations in one of the two genes that encode the $\alpha$ chains of type I collagen. Type I collagen, the main component of bone matrix, consists of two pro- $\alpha 1$ chains and one pro- $\alpha 2$ chain, which are encoded by COL1A1 (MIM \#120150; accession\# for mRNA Z74615.1) and COL1A2 (MIM \#120160; accession\# for mRNA Z74616.1) genes, respectively. COL1A1 is fixed on chromosome $17 \mathrm{q} 21.3-17 \mathrm{q} 22$ and COL1A2 on chromosome $7 \mathrm{q} 22.1$, their total lengths are 18 and $38 \mathrm{~kb}$, respectively, containing $\sim 50$ exons. Furthermore, each $\alpha$ chain consists of 1,014 amino acids forming a triple helical domain encoded by 43 exons. The folding of the three chains, with limited space, with triple helical domain restricts every third residue to a Gly, generating a repeating (Gly-X-Y)n sequence pattern. Once Gly in triple helix domains is substituted with other amino acids, the structure of type I collagen is destroyed. Gly is significant role in the formation of the triple helical domain, these sites are also the molecular basis of numerous diseases.

A number of gene mutations associated with COL1A1 and COL1A2 have been reported resulting in OI. To date, hundreds of mutations referring to $C O L 1 A 1$ and $C O L 1 A 2$ have been submitted to the University of Leicester's (Leicester, UK) database (http://www.le.ac.uk/genetics/collagen/) highlighting mutations of the collagen gene (6). A Chinese patient with OI type I disease, who possessed a novel single base substitution mutation (c.3263G $>\mathrm{A})$ in the COL1A1 gene was observed. This identification is likely to add to the the mutation database of collagen genes and provide more abundant materials for clinical diagnosis of heritable diseases and aid in the development of gene therapy and molecular diagnosis in the antepartum period.

\section{Materials and methods}

Case presentation and analysis. A 15-year-old Chinese male (Fig. 1) came to the Jinling Hospital (Nanjing, China) for genetic counseling of constitutional bone disease, which was 


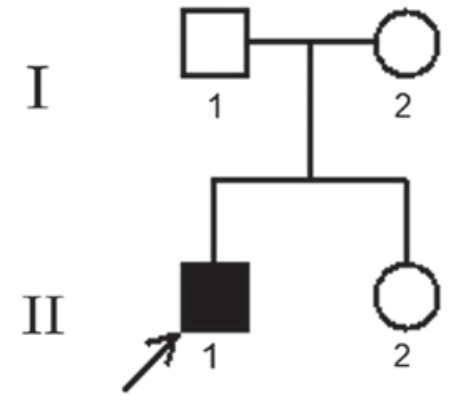

Figure 1. Pedigree of osteogenesis imperfecfa type I in a Chinese patient The black symbol indicates the patient with OI and white symbols represent unaffected individuals in the family. OI, osteogenesis imperfecta.

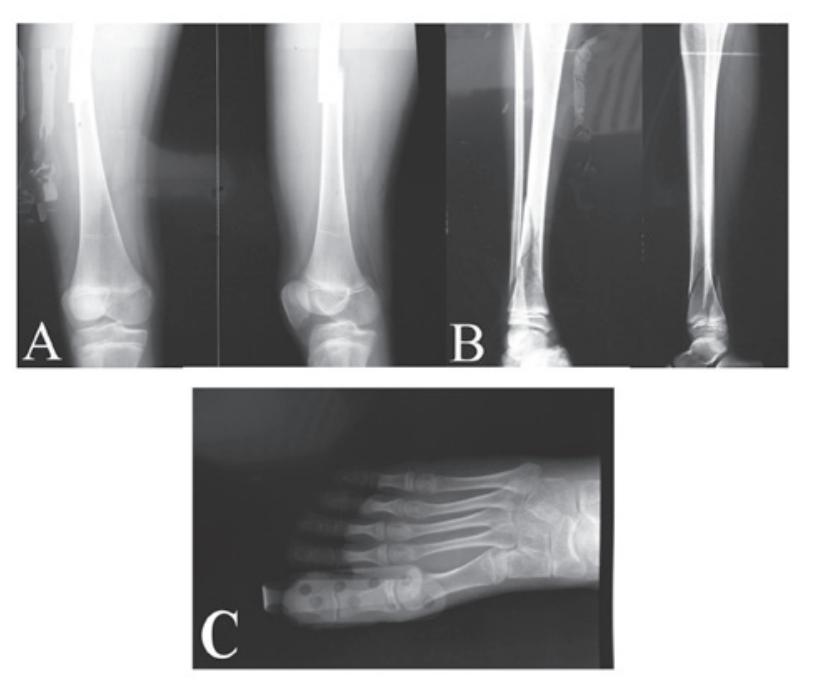

Figure 2. X-ray images from the patient with osteogenesis imperfecta. The patient previously suffered with severe fractures and a foot dislocation. There existed evidence of $>6$ previous limb fractures. (A) The femur fracture occurred on the right side following falling down the stairs in 2009 and (B) evidence of a previous comminuted fracture was observed on the right tibiofibula that occured without apparent stress in 2006 (the left radiographs of $\mathrm{A}$ and $\mathrm{B}$ were the adem position view and the right radiographs were the lateral position view) (C) The first apotelus dislocation occurred in 2010 and was the latest affection.

characterized by blue sclera and mild abnormalities with the teeth. The patient was $171 \mathrm{~cm}$ tall and weighed $54 \mathrm{~kg}$, with normal vision, audition and intelligence. According to the patient, he had previously suffered six fractures in the four limbs. Among these, foot dislocation was the latest fracture in 2010, and the most serious was a comminuted fracture in the right tibiofibula in 2006 (Fig. 2). The patient's parents and sister were all healthy. In addition, 250 healthy adults were used as controls.

DNA sequencing. A peripheral blood sample $(5 \mathrm{ml})$ was obtained from the patient, the patient's parents and sister, and 250 normal adults, respectively. All instructions were performed by the Institutional Review Board and the Committee on Ethics of Research Involving Human Subjects of Nanjing University Medical Center, Nanjing, China (7). The Wizard $^{\text {TM }}$ Genomic DNA Purification kit (Promega Corporation, Madison, WI, USA) was used to extract leucocyte genomic DNA, which was stored at $-20^{\circ} \mathrm{C}$, according to

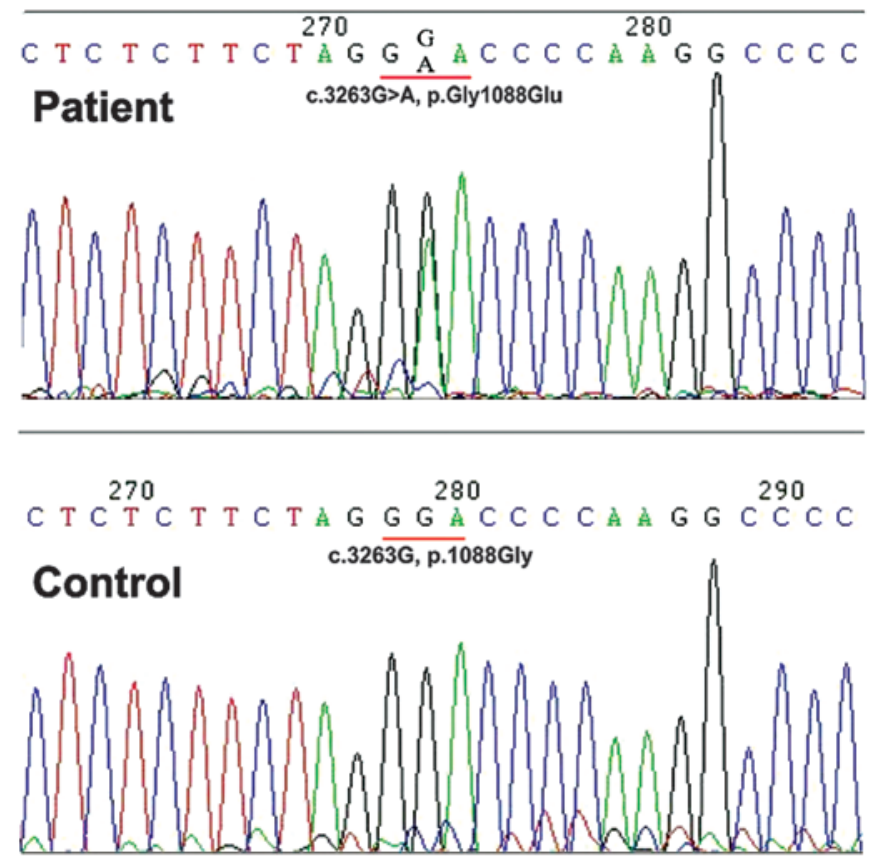

Figure 3. Sequence analysis of COL1A1 exon 45. The nucleotide sequence of the patient and a normal subjects are shown in the upper and lower panels, respectively. The patient has a novel heterozygous $\mathrm{G} \rightarrow \mathrm{A}$ mutation at nucleotide 3263 in exon 45 (c.3263G>A), resulting in the substitution of glycine with glutamic acid at codon 1088 (p.G1088E).

the manufacturer's instructions. The primers were designed according to $\sim 50$ exons of COL1A1 and COL1A2 (8), respectively, and polymerase chain reaction (PCR) was completed under the following conditions: $95^{\circ} \mathrm{C}$ for $5 \mathrm{~min}$ followed by 35 cycles of $95^{\circ} \mathrm{C}$ for $30 \mathrm{sec}, 56^{\circ} \mathrm{C}$ for $30 \mathrm{sec}$, and $72^{\circ} \mathrm{C}$ for $60 \mathrm{sec}(8)$. PCR products were sequenced directly using an ABI Prism 3700 automated sequencer (Applied Biosystems, Foster City, CA, USA). The sequencing results were compared with the NCBI Reference Sequence (NM_000088).

PCR-sequence specific primer (SSP) experiments. Allele-specific primer extension was used to confirm the heterozygous state. The wild-type gene sequence was amplified with primer A (5'-TGTCGCTTTTTC TCTTGTAGGG-3') plus primer C (5'-CTCGCATAATCGCTCTTTGTGTA-3') and the mutation sequence was amplified with primer B (5'-T GTCGCTTTTTCTCTTGTAGGA-3') plus primer C. It was expected that the products of wild-type and mutation-type sequences generated a fragment of $315 \mathrm{bp}$. The PCR cycling products were visualized using a UV transilluminator (WD-9403B; Beijing Liuyi Instrument Factory, Beijing, China) in a $2.0 \%$ agarose gel following electrophoresis and staining with ethidium bromide.

\section{Results}

A single base substitute mutation, c. $3263 \mathrm{G}>\mathrm{A}$ (p. Gly1088Glu), was identified in the sequence analysis of the COL1Al gene. The number of this mutation site was based on the cDNA sequence found in GenBank for human mRNA pre-pro- $\alpha$ I (I) (GenBank accession no. Z74615.1), using the A of ATG translation initiation start site as nucleotide +1 (9). 


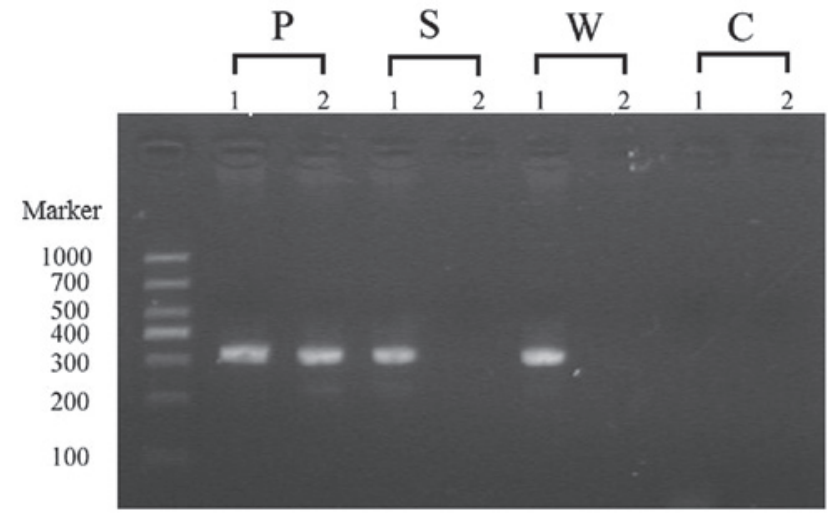

Figure 4. The electrophoretogram of PCR-SSP results of the affected and unaffected individuals. Each individual was amplified with two pairs of primers. Lane 1, represents PCR product amplified with primer A plus C, which were amplified in homozygotes and heterozygotes. Lane 2, represents PCR product amplified with primer A plus B, which was amplified only in heterozygotes. PCR, polymerase chian reaction; P, patient; S, sister; W, one of 250 normal adults; $\mathrm{C}$, negative control.

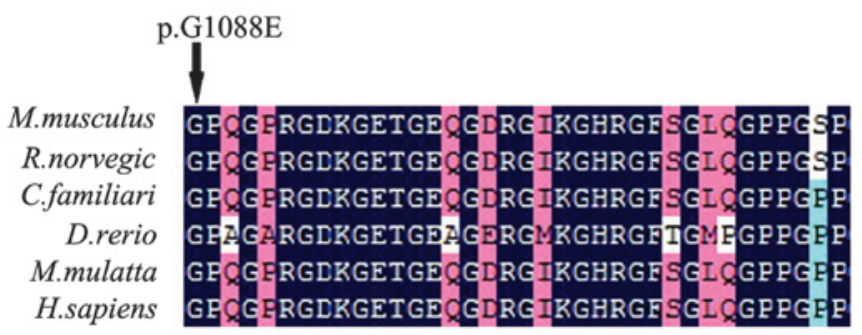

Figure 5. Multispecies alignment of the type I collagen $\alpha 1$ chain sequence shows that the mutation site (p.G1088E) indicated with an arrow, identified in the sporadic patient alters amino acids that are highly conserved in all species listed.

This mutation (c.3263G $>A$ ) was shown in the spectrum of the group (Fig. 3), the same mutation was not identified in the patient's parents or sister, or the 250 unrelated controls. The novelty of this mutation was identified, which was not defined in the Type I Collagen Mutation Database (http://www. le.ac.uk/genetics/collagen/), or the Human Gene Mutation Database (http://www.uwcm.ac.uk/uwcm/mg/hgmd().html). This mutation was further confirmed by PCR-SSP in the schematic diagram (Fig. 4). There also existed a single-nucleotide polymorphism in exon 33 (c.2298T >C) in this sporadic case, no other mutations were identified.

\section{Discussion}

There are numerous mutations in the prolyl 3-hydroxylation complex, including CRTAP (MIM \#605497), LEPRE1 (MIM \#610339) and PPIB (MIM \#123841), and the non-collagen related genes, FKBP10 (MIM \#607063), PLOD2 (MIM \#601865), SERPINF1 (MIM \#172860), SERPINH1 (MIM \#600943) and SP7 (MIM \#606633). These lead to defective bone development and abnormal collagen helix formation associated with a clinical spectrum of recessive OI (10). In addition, type I-IV of OI were almost all triggered by mutations in COL1A1 and COL1A2. Type I collagen, encoded by COLIA1 and COL1A2, consists of two pro- $\alpha 1$ chains and one pro- $\alpha 2$ chain. Each $\alpha$ chain includes an $\mathrm{N}$ - and C-terminal, and a core triple helical domain of the Gly-X-Y triplet repeat unit, which is highly conserved. The C-terminal is used for recognition and assembly of type I collagen $(11,12)$, and is cleaved until the triple helical domain is assembled into type I collagen. The N-terminal domain is also cleaved. According to the protein sequences in NCBI, with the reference sequence NM_000088.3, there are 1442 amino acids in the collagen pro- $\alpha 1$ (I) chain. The N-terminal domain consists of approximately the first 200 amino acids and the C-terminal domain consists of almost all the last 200 amino acids. (13). The remaining amino acids form the triple helix domain. Substitution mutations in the first 200 amino acids, occur only in the N-terminal domain, thus are nonlethal and exhibit a variable outcome. The triple helix domain is not considered as a domain purely, but rather as being composed of subdomains, each having its own specific function. The sizes of these subdomains remain unknown; however, the biochemical features of the substitution amino acids are linked to the role of the regions where the mutations occur.

The patient with OI type I had a guanine to adenine transition at nucleotide c. $3263 \mathrm{G}>\mathrm{A}$ in exon 45 that may result in a Gly to Glu substitution, which corresponds to the glycine position of Gly-X-Y repeat units within the triple helix domain of the collage protein (14). This variant was not identified in the patient's parents or sister, or 250 controls. As shown in an alignment of COL1A1 orthologs from six species of M. musculus, $R$. norvegicus, $C$.familiaris, D. rerio, $M$. mulatta, and $H$. sapiens, the original mutation position in COL1A1 is highly conserved across a number of species (Fig. 5). That suggests a substitution for Gly across the triple helix domain may induce different types of OI regardless of the substitution. However, the collagen gene mutations were not located in already existing hot spots.

Maurizia et al (15) reported a proband with disease of a lethal form of OI, in which an 18-month-old female succumbed to respiratory failure. The study showed that a $G$ to $\mathrm{C}$ conversion at nucleotide 3263 of pro- $\alpha 1$ (I) (c.3263G $>C$ ), which may change Gly to Ala in the triple helix domain. The mutation site was the same as that reported in the current study, however resulted in substitution with a different amino acid. The triple helix chain bearing these point mutations may exhibit secretion difficulties and the extensive mutable trimers may obstruct the secretion of normal chains. Furthermore, the chains containing one or two mutations exhibited poor thermal stability. The different substituted amino acids were hypothesized to result in a number of abnormalities, including collagen recognition, anomalous assembly and functional abnormality. To the best of our knowledge, this is the first this is the first study to demonstrate that disparate substitutions of amino acids at the same position may result in entirely different phenotypes from mild to lethal. It provided evidence for further establishment of phenotype-genotype associations, and it also suggested the foundations for the determination of the molecular mechanism underlying the development of OI.

In conclusion, a novel Gly substitution mutation (c.3263. $\mathrm{G}>\mathrm{A}$ ) in exon 45 of COLIAl gene resulting in OI type I was identified in a sporadic patient. The detailed molecular 
and clinical features are likely to be useful for extending the evidence for genetic and phenotypic heterogeneity and exploring the phenotype-genotype correlations in OI.

\section{Acknowledgements}

This study was supported by the National Natural Science Foundation of China (grant no. 30901652), the Key foundation of Jiangsu Science and Technology Bureau (grant no. BM2013058) and the Foundation of Jiangsu province (grant no. BK2011660). The authors would like to thank all members of the family for their cooperation in the study.

\section{References}

1. Byers PH: Disorders of collagen biosynthesis and structure. In: The Metabolic and Molecular Basis of Inherited Disease. Scriver CR, Beaudet AL, Sly WS and Valle D (eds). 7th edition. McGraw-Hill, New York, NY, pp4029-4077, 1995.

2. Byers PH, Wallis GA and Willing MC: Osteogenesis imperfecta: translation of mutation to phenotype. J Med Genet 28: 433-442, 1991.

3. Pollitt R, McMahon R, Nunn J, et al: Mutation analysis of COL1A1 and COL1A2 in patients diagnosed with osteogenesis imperfecta type I-IV. Hum Mutat 27: 716, 2006.

4. Morello R, Bertin TK, Chen Y, et al: CRTAP is required for prolyl 3-hydroxylation and mutations cause recessive osteogenesis imperfecta. Cell 127: 291-304, 2006.

5. Van Dijk FS, Nesbitt IM, Zwikstra EH, et al: PPIB mutations cause severe osteogenesis imperfecta. Am J Hum Genet 85: 521-527, 2009
6. Marini JC, Forlino A, Cabral WA, et al: Consortium for osteogenesis imperfecta mutations in the helical domain of type I collagen: regions rich in lethal mutations align with collagen binding sites for integrins and proteoglycans. Hum Mutat 28: 209-221, 2007

7. Xia XY, Cui YX, Huang YF, et al: A novel RNA-splicing mutation in COL1A1 gene causing osteogenesis imperfecta type I in a Chinese family. Clin Chim Acta 398: 148-151, 2008.

8. Körkkö J, Ala-Kokko L, De Paepe A, Nuytinck L, Earley J and Prockop DJ: Analysis of the COL1A1 and COL1A2 genes by PCR amplification and scanning by conformation-sensitive gel electrophoresis identifies only COL1A1 mutations in 15 patients with osteogenesis imperfecta type I: identification of common sequences of null-allele mutations. Am J Hum Genet 62: 98-110, 1998.

9. Lee KS, Song HR, Cho TJ, et al: Mutational spectrum of type I collagen genes in Korean patients with osteogenesis imperfecta. Hum Mutat 27: 599, 2006.

10. Forlino A, Cabral WA, Barnes AM and Marini JC: New perspectives on osteogenesis imperfecta. Nat Rev Endocrinol 7: 540-557, 2011.

11. Boot-Handford RP and Tuckwell DS: Fibrillar collagen: the key to vertebrate evolution? A tale of molecular incest. Bioessays 25: 142-151, 2003.

12. Exposito JY, Cluzel C, Garrone R and Lethias C: Evolution of collagens. Anat Rec 268: 302-316, 2002.

13. Stover DA and Verrelli BC: Comparative vertebrate evolutionary analyses of type I collagen: potential of COL1al gene structure and intron variation for common bone-related diseases. Mol Biol Evol 28: 533-542, 2011.

14. Weng YC, Sonni A, Labelle-Dumais C, et al: COL4A1 mutations in patients with sporadic late-onset intracerebral hemorrhage. Ann Neurol 71: 470-477, 2012.

15. Valli M, Sangalli A, Rossi A, et al: Osteogenesis imperfecta and type I collagen mutations A lethal variant carsed by a Gly910 - Ala substitution in the $\alpha 1(\mathrm{I})$ chain. Eur J Biochem 211: 415-419, 1993. 